\title{
Effect of peritoneal lavage with ulinastatin on the expression of NF- $\kappa B$ and TNF- $\alpha$ in multiple organs of rats with severe acute pancreatitis
}

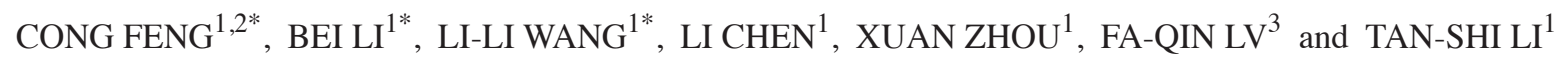 \\ ${ }^{1}$ Department of Emergency, PLA General Hospital, Beijing 100853; ${ }^{2}$ Li-Shi Road Outpatient Department, \\ Second Artillery General Hospital of the PLA, Beijing 100820; ${ }^{3}$ Department of Ultrasound, \\ PLA General Hospital, Beijing 100853, P.R. China
}

Received August 30, 2014; Accepted June 22, 2015

DOI: $10.3892 /$ etm.2015.2802

\begin{abstract}
The aim of the present study was to investigate the effect of peritoneal lavage with ulinastatin on the expression levels of nuclear factor $\kappa \mathrm{B}(\mathrm{NF}-\kappa \mathrm{B})$ and tumor necrosis factor (TNF)- $\alpha$ in multiple organs of rats with severe acute pancreatitis (SAP). Male Wistar rats were randomly divided into the following groups: Sham-operated (C), SAP model (SAP), saline lavage (SL), intravenous ulinastatin (IU) and peritoneal lavage with ulinastatin (UL). The SAP model was induced by the retrograde infusion of 5\% sodium taurocholate into the biliopancreatic ducts of the rats. Intraperitoneal lavage or injection was performed immediately following the establishment of the SAP model in groups SL, IU and UL. Intraperitoneal lavage with or without ulinastatin was performed for $3 \mathrm{~h}$. The survival time of half of the rats in each group was recorded over a 12-h period. At $3 \mathrm{~h}$ after the induction of SAP, inflammatory mediators and the expression levels of NF- $\kappa \mathrm{B}$ and TNF- $\alpha$ in multiple organs of the rats in each group were also detected. The survival rates of the rats in group UL at $6 \mathrm{~h}$ and $9 \mathrm{~h}$ were increased compared with those in group SAP, and were also higher than that in groups SL and IU. The levels of serum inflammatory mediators were effectively reduced in groups SL, IU and UL, the greatest effects were observed in group UL. The expression levels of $\mathrm{NF}-\kappa \mathrm{B}$ and TNF- $\alpha$ in multiple organs were significantly lower in group UL compared with other groups. Intraperitoneal
\end{abstract}

Correspondence to: Dr Tan-Shi Li, Department of Emergency, PLA General Hospital, 28 Fuxing Road, Beijing 100853, P.R. China E-mail: 1ts301@sohu.com

Dr Fa-Qin Lv, Department of Ultrasound, PLA General Hospital, 28 Fuxing Road, Beijing 100853, P.R. China

E-mail:1vjin8912@163.com

*Contributed equally

Key words: severe acute pancreatitis, intraperitoneal lavage, ulinastatin, NF- $\mathrm{KB}, \mathrm{TNF}-\alpha$ lavage with ulinastatin significantly ameliorated the inflammatory reaction and inhibited NF- $\mathrm{KB}$ and $\mathrm{TNF}-\alpha$ expression in multiple organs of SAP model rats.

\section{Introduction}

Severe acute pancreatitis (SAP) is a serious systemic inflammatory disease with high mortality rate, which may cause systemic inflammatory response syndrome (SIRS) and multiple organ dysfunction syndrome (MODS) due to excessive inflammatory reactions. However, the exact pathogenesis remains unclear (1). Studies have found that nuclear factor $\kappa \mathrm{B}(\mathrm{NF}-\kappa \mathrm{B})$ has a high level of expression in SAP, and plays an important role in regulating the inflammatory response $(2,3)$. Tumor necrosis factor (TNF)- $\alpha$ is an inflammatory mediator that is also plays a critical role in the pathogenesis of acute pancreatitis by driving the subsequent inflammatory response (4). Ulinastatin is a purified antiprotease isolated from the fresh urine of healthy adults, which possesses powerful anti-inflammatory effects and has been demonstrated to exert significant therapeutic effects in several forms of acute pancreatitis $(5,6)$. However, the impact of ulinastatin administered by peritoneal lavage on the expression levels of NF- $\mathrm{BB}$ and TNF- $\alpha$ in multiple organs in SAP has not been studied. According to our preliminary study (7), peritoneal lavage with the addition of ulinastatin at a concentration of $62.5 \mathrm{U} / \mathrm{ml}$ to the lavage fluid exerts the best therapeutic effect. The present study investigated the expression levels of $\mathrm{NF}-\kappa \mathrm{B}$ and $\mathrm{TNF}-\alpha$ in multiple organs and thereby explored the underlying mechanism of intraperitoneal lavage with ulinastatin on a rat model of SAP. The results may provide convincing theoretical evidence supporting the further experimental and clinical application of ulinastatin as a peritoneal lavage.

\section{Materials and methods}

Experimental animals and ethics. A total of 100 healthy male Wistar rats weighing $300 \pm 15 \mathrm{~g}$ were obtained from the Experimental Animal Center of the PLA General Hospital (Beijing, China). The experimental protocol was approved by the Ethics Committee for Animal Research from the PLA 
General Hospital. Ethical standards were adhered to and the rats received humane care.

Reagents. Reagents were purchased as follows: Chloral hydrate (Shanghai Yingxin Laboratory Equipment Co., Ltd., Shanghai, China); sodium taurocholate (Shanghai Hufeng Biotechnology Co., Ltd., Shanghai, China); ulinastatin (Guangdong Tianpu Biochemical Pharmaceutical Co., Ltd., Guangzhou, China); IL-1 and IL-6 assay kits (Shanghai Hengyuan Biological Technology Co., Ltd., Shanghai, China); and rat soluble NF- $\kappa B$ and TNF- $\alpha$ ELISA kits (Wuhan Hua Mei Biological Engineering Co., Ltd., Wuhan, China).

Experimental groups. Rats were randomly divided into five groups: Group C $(\mathrm{n}=20)$, sham-operated without induction of SAP, peritoneal lavage or intravenous injection, but with insertion of a catheter; group SAP $(n=20)$, induction of SAP without peritoneal lavage or intravenous injection, but with insertion of a catheter; group SL ( $\mathrm{n}=20)$, saline lavage for $3 \mathrm{~h}$ immediately after the induction of SAP; group IU $(n=20)$, intravenous ulinastatin at 2,500 U/100 g immediately after the induction of SAP and with insertion of a catheter, but without peritoneal lavage; group UL $(\mathrm{n}=20)$, ulinastatin lavage at a concentration of $62.5 \mathrm{U} / \mathrm{ml}$ for $3 \mathrm{~h}$ immediately after the induction of SAP.

Animal model. All rats fasted for $12 \mathrm{~h}$ and had no water for $4 \mathrm{~h}$ prior to surgery. Rats were anesthetized with intraperitoneal injections of $10 \%$ chloral hydrate $(3 \mathrm{ml} / \mathrm{kg})$. After making an incision in the abdomen, clamping the opening and remote part of the duodenal bile duct with injury-free metal clips, a syringe needle was inserted into the opening of the duodenal bile duct. Then, 5\% sodium taurocholate (freshly prepared in saline solution, $0.6 \mathrm{ml}$ ) was retrogradely injected into the bile duct at constant rate of $0.2 \mathrm{ml} / \mathrm{min}$ using an infusion pump. After $5 \mathrm{~min}$, the needle and metal clips were removed. This procedure produces a consistently high mortality rate (>80\% within $12 \mathrm{~h}$ ) (8). Group C was sham-operated without induction of SAP.

Prior to closure of the abdomen, a silicon catheter (catheter A) with five lateral outlets was placed adjacent to the pancreas and another silicon catheter (catheter B) with five lateral outlets was placed in the pelvic cavity. All groups accepted peritoneal catheter insertion.

Peritoneal lavage. Intraperitoneal lavage was performed immediately following the establishment of the SAP model in the rats of groups SL and UL. Lavage fluid was warmed to $37^{\circ} \mathrm{C}$ and injected from catheter $\mathrm{A}$ at $80 \mathrm{ml} / \mathrm{h}$ for $15 \mathrm{~min}$ and catheter B was blocked. Then, catheter A was blocked and the fluid was allowed to flow out for $15 \mathrm{~min}$ from catheter B. Thus, each lavage procedure lasted $30 \mathrm{~min}$, and lavage was performed 6 times ( $3 \mathrm{~h}$ total lavage time). Volume input and output were monitored. The lavage fluid consisted of saline solution with or without the addition of ulinastatin at a concentration of $62.5 \mathrm{U} / \mathrm{ml}$, which our earlier study indicated to exert the optimum therapeutic effect. Following the lavage procedure, the catheters A and B were blocked and the rats were kept in single cages, with free access to water and no solid food.

Intravenous ulinastatin. To compare the effect of peritoneal lavage with that of intravenously administered ulinastatin, group IU immediately received intravenous ulinastatin $2,500 \mathrm{U} / 100 \mathrm{~g}$ (freshly prepared in $0.15 \mathrm{ml}$ saline solution, which approximates the total dose in group UL) from the caudal vein after SAP induction, but no lavage.

Observation indices. The survival time of each group $(\mathrm{n}=10$ for each) was recorded for $12 \mathrm{~h}$ and the survival rate at 3,6 , 9 and $12 \mathrm{~h}$ was calculated. Rats surviving to $12 \mathrm{~h}$ were anesthetized and sacrificed humanely. At $3 \mathrm{~h}$ after surgery, rats in each group ( $\mathrm{n}=10$ for each) were also humanely sacrificed, and blood samples were collected for the detection of inflammatory mediators (including IL-1 and IL-6), and specimens of multiple organs (including pancreas, lung, liver and kidney) were prepared for determination of the expression levels of $\mathrm{NF}-\mathrm{\kappa B}$ and TNF- $\alpha$.

All tissues were homogenized with cold saline at a 1:9 dilution using a PowerGen 1000 homogenizer (Thermo Fisher Scientific, Waltham, MA, USA). All tissue specimens were rinsed in cold saline to remove blood, dried with filter paper, cut into small pieces using a disposable scalpel, and $0.4 \mathrm{~g}$ tissue (pancreas, lung, live or kidney) was added to a $10-\mathrm{ml}$ beaker. Next, $3.6 \mathrm{ml}$ cold saline was added to the tissue and homogenization was achieved by using the homogenizer for 1-2 min. Subsequently, $10 \%$ homogenate was prepared using low temperature centrifugation at $1,000 \mathrm{x}$ g for $10-15 \mathrm{~min}$ at $4^{\circ} \mathrm{C}$ and the supernatant was obtained for determination.

Assays for serum IL-1, IL-6 content and supernatant of homogenate $N F-\kappa B$ and TNF- $\alpha$ expression levels. Samples for IL-1, IL-6, NF- $\kappa B$ and TNF- $\alpha$ were aliquoted in portions and stored at $-80^{\circ} \mathrm{C}$ for no longer than 2 months. Measurements were performed using the IL-1, IL-6, rat soluble NF- $\kappa B$ and TNF- $\alpha$ ELISA kits and a standard ELISA reader according to the manufacturer's instructions.

Statistical analysis. Data are expressed as mean \pm standard deviation for normally distributed variables or median and interquartile range for highly skewed variables. Statistical analyses were performed using the SPSS software package, version 19.0 (SPSS, Inc., Chicago, IL, USA).

In survival experiments, the survival rate after the $12 \mathrm{~h}$ observation period was conducted by the Kaplan-Meier or Kruskal-Wallis $\mathrm{H}$ test. Analysis of variance was used for comparison of normally distributed data. Multiple comparisons were subjected to Kruskal-Wallis $\mathrm{H}$ test and Bonferroni correction test. The Chi-square test was used to evaluate the equality of frequencies for discrete variables. $\mathrm{P}<0.05$ was considered to indicate a statistically significant difference.

\section{Results}

Survival rate. All rats in group C were alive at $12 \mathrm{~h}(100 \%)$. At $6 \mathrm{~h}$, group SAP had a survival rate of $20 \%$ and the survival rates in groups SL (50\%), IU (60\%) and UL (100\%) were all increased compared with that in group SAP. However, at $9 \mathrm{~h}$, only group UL $(50 \%)$ had a greatly increased survival rate compared with that in than groups SAP (10\%), SL (20\%) and IU $(20 \%)$. At $12 \mathrm{~h}$, there was no difference in survival rate among groups SAP $(0 \%)$, SL (0\%), IU (0\%) and UL (10\%). The results are summarized in Table I. 
Table I. Comparison of survival rate among the five groups (each $\mathrm{n}=10$ ).

\begin{tabular}{|c|c|c|c|c|c|}
\hline \multirow[b]{2}{*}{ Time point } & \multicolumn{5}{|c|}{ Survival rate $(\%)$} \\
\hline & $\mathrm{C}$ & SAP & SL & $\mathrm{IU}$ & UL \\
\hline $3 \mathrm{~h}$ & $10 / 10(100)$ & $9 / 10(90)$ & $10 / 10(100)$ & 10/10 (100) & $10 / 10(100)$ \\
\hline $6 \mathrm{~h}$ & $10 / 10(100)$ & $2 / 10(20)^{\mathrm{a}}$ & $5 / 10(50)^{\mathrm{a}}$ & $6 / 10(60)^{a, b}$ & $10 / 10(100)^{b}$ \\
\hline $9 \mathrm{~h}$ & $10 / 10(100)$ & $1 / 10(10)^{\mathrm{a}}$ & $2 / 10(20)^{\mathrm{a}}$ & $2 / 10(20)^{\mathrm{a}}$ & $5 / 10(50)^{\mathrm{b}}$ \\
\hline $12 \mathrm{~h}$ & $10 / 10(100)$ & $0 / 10(0)^{\mathrm{a}}$ & $0 / 10(0)^{\mathrm{a}}$ & $0 / 10(0)^{\mathrm{a}}$ & $1 / 10(10)^{\mathrm{a}}$ \\
\hline
\end{tabular}

${ }^{\text {a }}<<0.05$ compared with group $\mathrm{C}$; ${ }^{\mathrm{b}}<0.05$ compared with group SAP. C, control; SAP, severe acute pancreatitis; SL, saline lavage; IU, intravenous ulinastatin; UL, ulinastatin lavage.

Table II. Effect of the different treatments on inflammatory mediators.

\begin{tabular}{lccrrr}
\hline Mediator & C & SAP & SL & IU & UL \\
\hline IL-1 (ng/ml) & $0.047 \pm 0.016$ & $\begin{array}{r}0.182 \pm 0.047^{\mathrm{a}} \\
129.15 \pm 35.85^{\mathrm{a}}\end{array}$ & $\begin{array}{r}0.102 \pm 0.027^{\mathrm{a}, \mathrm{b}} \\
111.35 \pm 20.38^{\mathrm{a}}\end{array}$ & $\begin{array}{r}0.105 \pm 0.039^{\mathrm{a}, \mathrm{b}} \\
108.73 \pm 34.96^{\mathrm{a}, \mathrm{c}}\end{array}$ & $\begin{array}{l}0.071 \pm 0.028^{\mathrm{b}} \\
\text { IL-6 }(\mathrm{pg} / \mathrm{ml})\end{array}$ \\
\hline
\end{tabular}

Values are presented as mean \pm standard deviation. ${ }^{\mathrm{a}} \mathrm{P}<0.01$ vs. group $\mathrm{C}$; ${ }^{\mathrm{b}} \mathrm{P}<0.01,{ }^{\mathrm{c}} \mathrm{P}<0.05$ vs. group $\mathrm{SAP}$. $\mathrm{C}$, control; SAP, severe acute pancreatitis; SL, saline lavage; IU, intravenous ulinastatin; UL, ulinastatin lavage.
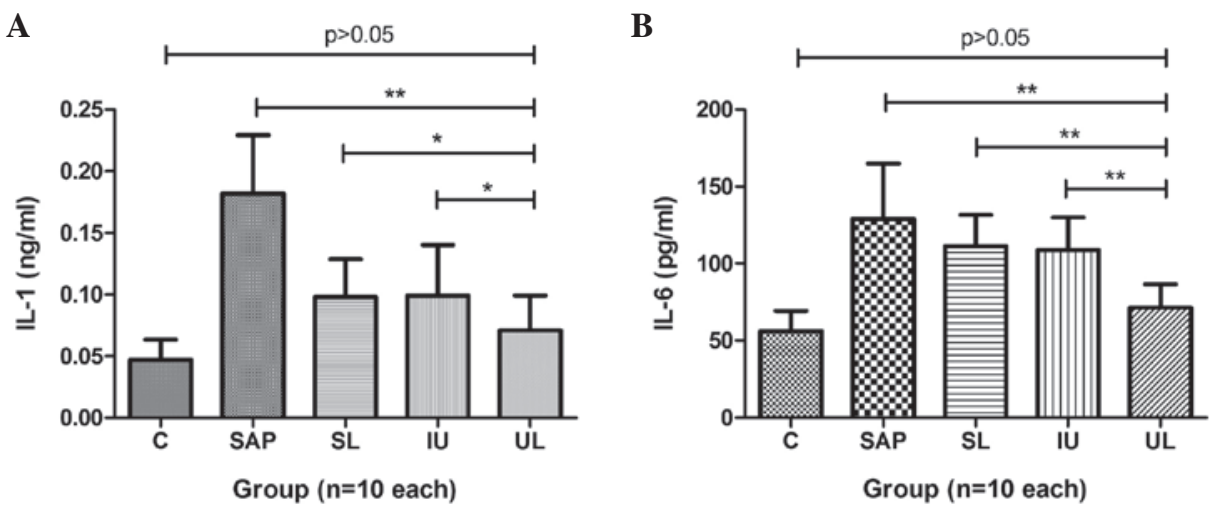

Figure 1. Effect of peritoneal lavage with ulinastatin on inflammatory mediators. The levels of (A) IL-1 and (B) IL-6 in group SAP were significantly increased compared with those in group C. Groups SL, IU and UL all exhibited reduced levels of IL-1 and IL-6 compared with those in group SAP. Moreover, group UL had lower levels of IL-1 and IL-6 than groups SL and IU. ${ }^{*} \mathrm{P}<0.05,{ }^{* *} \mathrm{P}<0.01$. C, control; SAP, severe acute pancreatitis; SL, saline lavage; IU, intravenous ulinastatin; UL, ulinastatin lavage; IL, interleukin.

Inflammatory mediators. The plasma levels of IL-1 and IL-6 in the rats of group SAP were significantly increased compared with those in group C. The levels of IL-1 in groups SL, IU and UL were significantly reduced compared with those in group SAP. The lowest levels of IL-1 were observed in group UL (Fig. 1). Compared with the IL-6 levels in group SAP, those in groups IU and UL were significantly reduced, and the strongest effect was observed in group UL, but group SL did not exhibit a statistically significant difference (Fig. 1). The results are summarized in Table II.

Expression levels of TNF- $\alpha$ in multiple organs. The expression levels of TNF- $\alpha$ in the pancreas, lung, liver and kidney of rats in group SAP were significantly increased compared with those in group C. In groups SL, IU and UL, the expression levels of TNF- $\alpha$ in the pancreas were significantly reduced compared with those in group SAP, and groups SL and UL exhibited greater reductions in TNF- $\alpha$ levels than group IU (Fig. 2A). The expression levels of TNF- $\alpha$ in the lungs of rats in groups SL, IU and UL were significantly reduced compared with those in group SAP, and the TNF- $\alpha$ levels in group UL were the lowest among these three groups (Fig. 2B). The expression levels of TNF- $\alpha$ in the livers of the rats in groups SL, IU and UL were significantly reduced compared with those in group SAP. The effects in groups UL and SL were comparable and stronger than those in group IU (Fig. 2C). The expression levels of TNF- $\alpha$ in the kidneys of the rats in groups SL, IU and UL were significantly reduced compared with those in group SAP, and were reduced to the greatest extent in group UL (Fig. 2D). The results are summarized in Table III. 
Table III. Effect of different treatment on the expression levels of TNF- $\alpha$ in multiple organs at $3 \mathrm{~h}(\mathrm{pg} / \mathrm{ml})$.

\begin{tabular}{|c|c|c|c|c|c|}
\hline Organ & $\mathrm{C}$ & SAP & SL & IU & UL \\
\hline Pancreas & $4.497 \pm 0.275$ & $12.153 \pm 0.398^{\mathrm{a}}$ & $5.535 \pm 0.260^{\mathrm{a}, \mathrm{b}}$ & $6.651 \pm 0.213^{\mathrm{a}, \mathrm{b}}$ & $5.287 \pm 0.334^{\mathrm{a}, \mathrm{b}}$ \\
\hline Lung & $11.126 \pm 1.513$ & $21.383 \pm 1.834^{\mathrm{a}}$ & $14.219 \pm 1.002^{\mathrm{a}, \mathrm{b}}$ & $15.670 \pm 1.614^{\mathrm{a}, \mathrm{b}}$ & $12.870 \pm 1.412^{\mathrm{b}, \mathrm{c}}$ \\
\hline Liver & $11.079 \pm 0.566$ & $25.054 \pm 0.605^{\mathrm{a}}$ & $12.797 \pm 0.842^{\mathrm{a}, \mathrm{b}}$ & $15.009 \pm 0.866^{\mathrm{a}, \mathrm{b}}$ & $12.929 \pm 0.745^{\mathrm{a}, \mathrm{b}}$ \\
\hline Kidney & $9.587 \pm 0.405$ & $14.566 \pm 0.355^{\mathrm{a}}$ & $10.802 \pm 0.538^{\mathrm{a}, \mathrm{b}}$ & $12.403 \pm 0.731^{\mathrm{b}, \mathrm{c}}$ & $10.082 \pm 0.436^{\mathrm{a}, \mathrm{b}}$ \\
\hline
\end{tabular}

Values are presented as mean \pm standard deviation. ${ }^{\mathrm{a}} \mathrm{P}<0.01$ vs. group $\mathrm{C}$; ${ }^{\mathrm{b}} \mathrm{P}<0.01$ vs. group $\mathrm{SAP} ;{ }^{\mathrm{c}} \mathrm{P}<0.05$ vs. group $\mathrm{C}$. $\mathrm{C}$, control; $\mathrm{SAP}$, severe acute pancreatitis; SL, saline lavage; IU, intravenous ulinastatin; UL, ulinastatin lavage; TNF- $\alpha$, tumor necrosis factor.

A

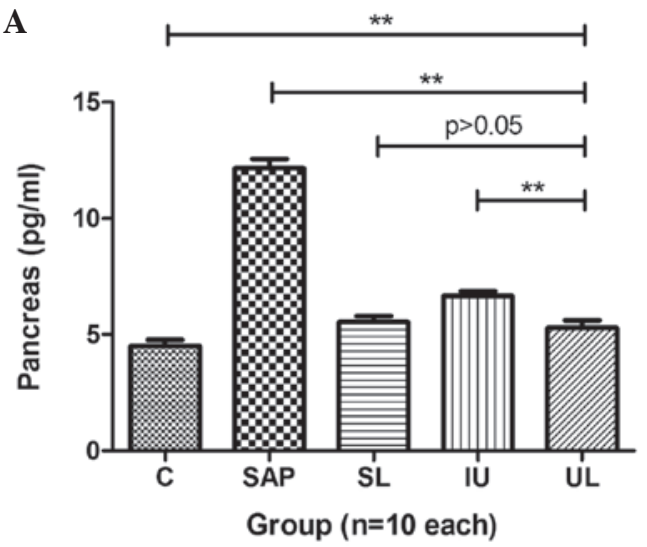

C

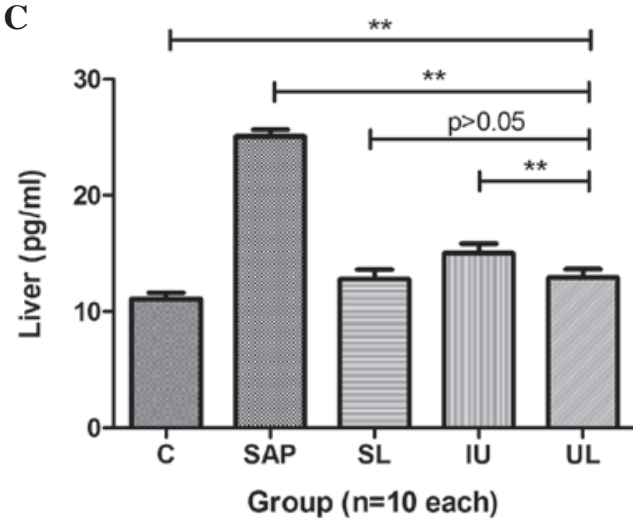

B

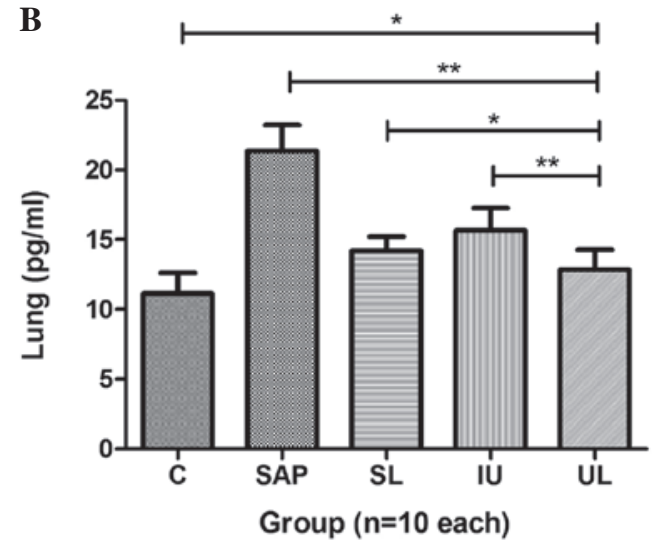

D

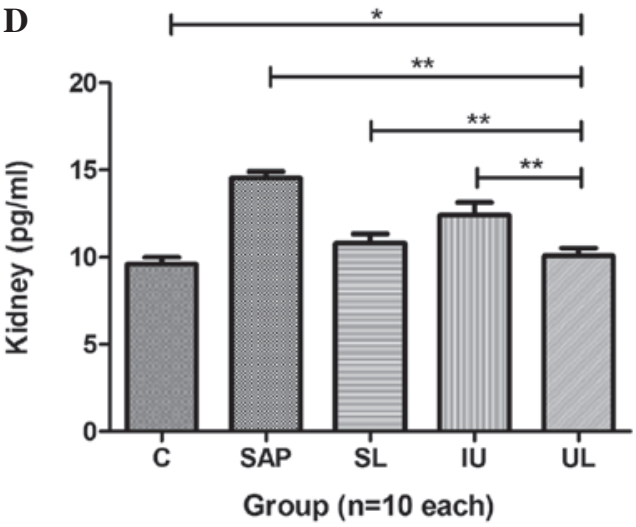

Figure 2. Comparison of different treatments on the expression levels of TNF- $\alpha$ in the (A) pancreas, (B) lung, (C) liver and (D) kidney. The expression levels of TNF- $\alpha$ in multiple organs were greatly increased in group SAP compared with those in group C. The treatments administered to groups SL, IU and UL all significantly decreased the expression levels of TNF- $\alpha$ in multiple organs compared with those in group SAP and group UL exhibited the greatest reduction in expression levels. ${ }^{*} \mathrm{P}<0.05,{ }^{* *} \mathrm{P}<0.01$. C, control; SAP, severe acute pancreatitis; SL, saline lavage; IU, intravenous ulinastatin; UL, ulinastatin lavage; TNF- $\alpha$, tumor necrosis factor- $\alpha$.

Expression levels of $N F-\kappa B$ in multiple organs. The expression levels of $\mathrm{NF}-\kappa \mathrm{B}$ in the pancreas, lung, liver and kidney of rats in group SAP were significantly greater than those in group $\mathrm{C}$. In groups SL and UL, the expression levels of NF- $\mathrm{KB}$ in the pancreas were significantly reduced compared with those in group SAP, but those of group IU were not. The effects observed in group SL were almost the same as those in group UL (Fig. 3A). The expression levels of $\mathrm{NF}-\kappa \mathrm{B}$ in the lungs of rats in groups SL, IU and UL were significantly reduced compared with those in group SAP, with group UL exhibited the greatest reduction (Fig. 3B). The expression levels of NF- $\mathrm{NB}$ in the livers of the rats in groups SL, IU and UL were significantly reduced compared with those in group SAP. The effects observed in groups UL and SL were greater than those in group IU (Fig. 3C). The expression levels of NF- $\kappa \mathrm{B}$ in the kidneys of rats in groups SL, IU and UL were significantly reduced compared with those in group SAP. The reduction in group UL was greater than that in groups SL and IU (Fig. 3D). The results are summarized in Table IV.

\section{Discussion}

SAP is a systemic disease with a high mortality rate, which may induce SIRS and MODS due to the excessive inflammatory reactions that play an important role in the pathogenesis of SAP. 


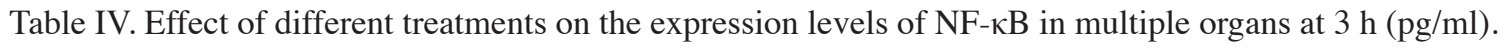

\begin{tabular}{lccccc}
\hline Organ & C & SAP & SL & IU & UL \\
\hline Pancreas & $3.198 \pm 0.295$ & $4.424 \pm 0.153^{\mathrm{a}}$ & $3.716 \pm 0.266^{\mathrm{a}, \mathrm{b}}$ & $4.254 \pm 0.166^{\mathrm{a}}$ & $3.574 \pm 0.297^{\mathrm{a}, \mathrm{b}}$ \\
Lung & $4.543 \pm 0.349$ & $6.821 \pm 0.276^{\mathrm{a}}$ & $5.215 \pm 0.300^{\mathrm{a}, \mathrm{b}}$ & $5.890 \pm 0.359^{\mathrm{a}, \mathrm{b}}$ & $4.856 \pm 0.285^{\mathrm{b}, \mathrm{c}}$ \\
Liver & $3.489 \pm 0.257$ & $6.250 \pm 0.384^{\mathrm{a}}$ & $3.781 \pm 0.276^{\mathrm{b}, \mathrm{c}}$ & $4.766 \pm 0.334^{\mathrm{a}, \mathrm{b}}$ & $3.793 \pm 0.223^{\mathrm{b}, \mathrm{c}}$ \\
Kidney & $2.690 \pm 0.277$ & $5.568 \pm 0.297^{\mathrm{a}}$ & $4.665 \pm 0.267^{\mathrm{a}, \mathrm{b}}$ & $5.225 \pm 0.325^{\mathrm{a}, \mathrm{d}}$ & $3.878 \pm 0.329^{\mathrm{a}, \mathrm{b}}$ \\
\hline
\end{tabular}

Values are presented as mean \pm standard deviation. ${ }^{\mathrm{a}} \mathrm{P}<0.01$ vs. group $\mathrm{C}$; ${ }^{\mathrm{b}} \mathrm{P}<0.01$ vs. group $\mathrm{SAP} ;{ }^{\mathrm{c}} \mathrm{P}<0.05$ vs. group $\mathrm{C}$; ${ }^{\mathrm{d}} \mathrm{P}<0.05$ vs. group $\mathrm{SAP}$. $\mathrm{C}$, control; SAP, severe acute pancreatitis; SL, saline lavage; IU, intravenous ulinastatin; UL, ulinastatin lavage; NF- $\kappa \mathrm{B}$, nuclear factor- $\kappa \mathrm{B}$.

A

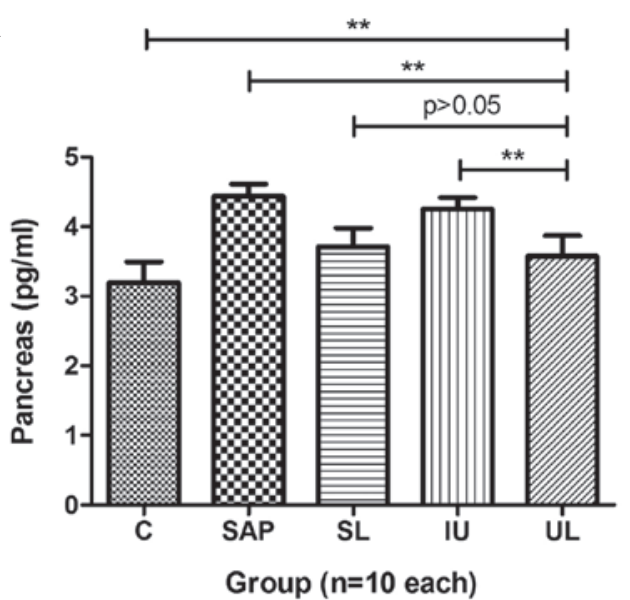

$\mathbf{C}$

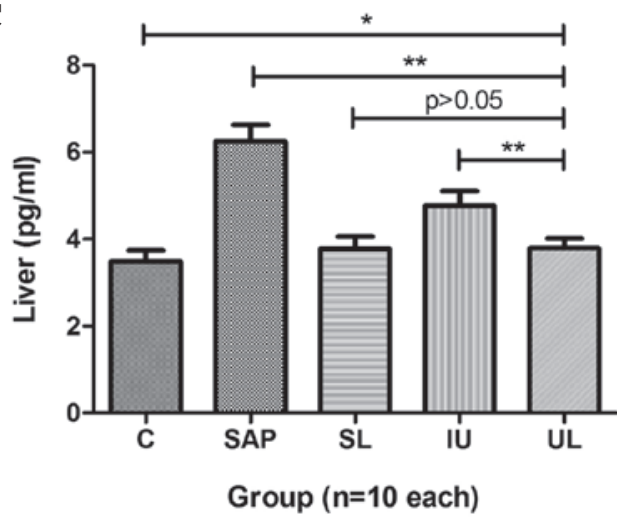

B

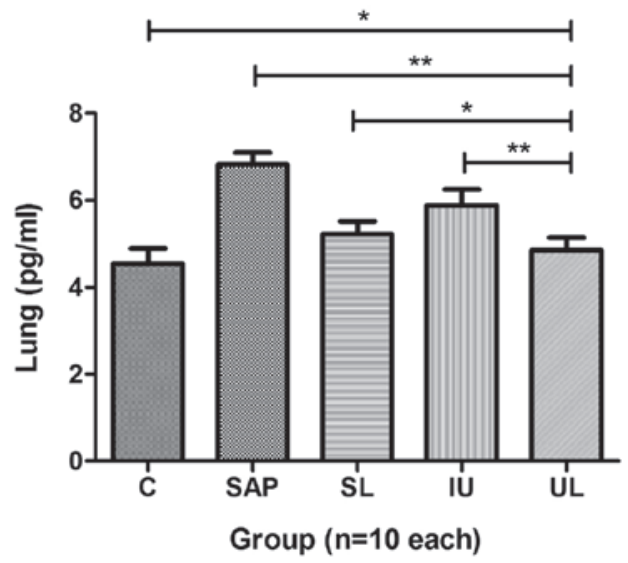

D

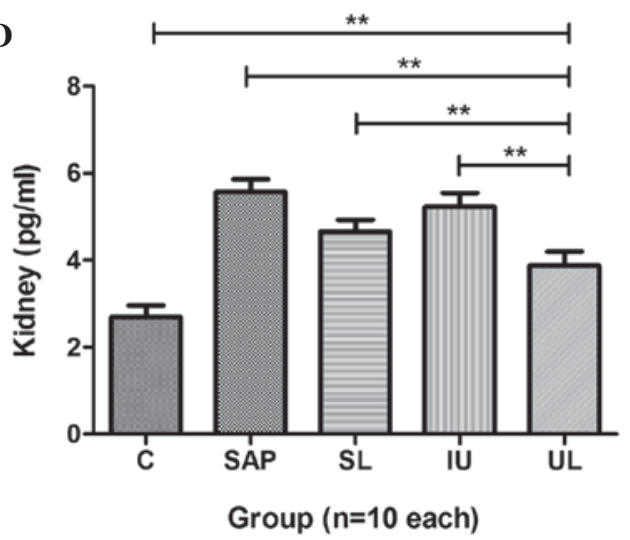

Figure 3. Comparison of different treatments on the expression levels of NF-kB in the (A) pancreas, (B) lung, (C) liver and (D) kidney. The expression levels

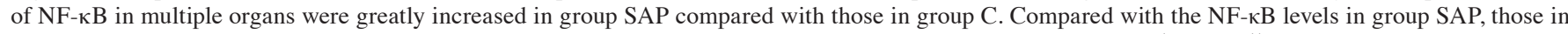
groups SL, IU and UL were significantly decreased and group UL exhibited the greatest reduction in NF-kB levels. ${ }^{*} \mathrm{P}<0.05,{ }^{* *} \mathrm{P}<0.01$. C, control; SAP, severe acute pancreatitis; SL, saline lavage; IU, intravenous ulinastatin; UL, ulinastatin lavage; NF-kB, nuclear factor-kB.

It has been reported that pancreatic enzymes released into the peritoneal exudate could aggravate the inflammatory condition by damaging multiple organs $(1,9)$. Therefore, peritoneal lavage and antiprotease therapy may bring an improved therapeutic effect through being applied locally to the peritoneal cavity (10). The glycoprotein ulinastatin is widely used to treat patients with SAP via the intravenous route $(11,12)$; one of its therapeutic functions is regulation of the inflammatory response. Ulinastatin may provide a greater therapeutic effect for SAP if administered by peritoneal lavage as only a small amount of intravenously administered ulinastatin is able to reach the pancreas and other organs because of microcirculatory dysfunction (13).
Studies of SAP treatment by ulinastatin-containing peritoneal lavage have rarely been reported. However, on the basis of our previous study, peritoneal lavage with the addition of $62.5 \mathrm{U} / \mathrm{ml}$ ulinastatin to the lavage fluid provides the optimum effect in the treatment of SAP. In the survival experiment conducted in the present study, group UL exhibited the greatest median survival rate among the four treatment groups at 6 and $9 \mathrm{~h}$. Ulinastatin administered intravenously or by peritoneal lavage improved the survival rate; however, peritoneal lavage with ulinastatin provided the greatest effect.

IL-1 and IL-6 are significant proinflammatory cytokines that are present at elevated levels in the pathogenesis of acute 
pancreatitis and play a critical role in mediating the systemic inflammatory response $(14,15)$. It has been reported that the levels of proinflammatory cytokines correlate with the severity of acute pancreatitis (16). The present study demonstrated that the serum levels of IL-1 and IL-6 in group SAP were significantly elevated compared with those in group C. Following treatment, the levels of IL-1 and IL- 6 in group UL were much lower than those in group SAP and were also lower than those in groups SL and IU. This indicated that the administration of ulinastatin by peritoneal lavage most effectively lowered the levels of inflammatory mediators in the serum, alleviated the severe systemic inflammatory response and inhibited the progress of SAP by inhibiting the release of inflammatory mediators.

TNF- $\alpha$ is considered to be one of the major factors associated with the multiple tissue or organ damage caused by a systemic inflammatory response and could be a therapeutic target for therapeutic applications in SAP (17). The results of the present study showed that the levels of TNF- $\alpha$ in multiple organs of group SAP were greater than those in group C. After treatment, the expression levels of TNF- $\alpha$ in the pancreas, lung, liver, and kidney of group UL were significantly lower than those in groups SL and IU. This indicated that saline lavage with ulinastatin protected multiple organs from further damage through inhibiting TNF- $\alpha$ to attenuate the systemic inflammatory response. The results for the serum levels of IL-1 and IL-6 in the present study are consistent with this.

A number of studies have demonstrated that the activation of $N F-\kappa B$ plays a significant role in the onset of SAP, due to its ability to regulate the expression of inflammatory mediators $(16,18)$. The activation of $N F-\kappa B$ has been shown to increase the expression of TNF- $\alpha$ (19). In the present study, following the induction of SAP, it was observed that $\mathrm{NF}-\kappa \mathrm{B}$ was activated significantly in multiple organs of the rats in group SAP compared with group $\mathrm{C}$; the expression of TNF- $\alpha$ in the various organs from group SAP also increased markedly. Peritoneal lavage with ulinastatin significantly reduced the expression of NF- $\kappa \mathrm{B}$ in multiple organs; among the groups SL, IU and UL, the greatest effects were observed in group UL. The trend of changes in TNF- $\alpha$ in multiple organs was similar to that of NF- $\kappa$ B among groups SAP, SL, IU and UL. Therefore, it can be inferred that peritoneal lavage with ulinastatin alleviates the inflammatory response and progression of SAP by inhibiting the activation of NF- $\mathrm{NB}$. This may lower the expression of TNF- $\alpha$, thereby reducing the release of IL- 1 and IL- 6 .

In conclusion, this study provides evidence that peritoneal lavage with ulinastatin may effectively improve the prognosis of SAP by mitigating the extent of inflammation, specifically by inhibiting $N F-\kappa B$ activation, lowering the expression of TNF- $\alpha$ and reducing the levels of IL-1 and IL- 6 . The results in this study may provide a basis for further experimental or clinical research into SAP.

\section{Acknowledgements}

The authors acknowledge the financial support provided by the Innovative Doctor Project of the PLA General Hospital and the Welfare Industry Research Program of the Ministry of Health: The establishment of new diagnostic criteria of severe pneumonia and acute lung injury and the optimization scheme of clinical treatment (No. 201302017) and the 'Twelfth Five Year Plan' in rural areas of China National Science and Technology Planning Issues (No. 2012BAJ18B02-03). The authors are also grateful to Professor Chong-Hui Li (Department of Hepatobiliary Surgery, PLA General Hospital) for her considerable recommendations.

\section{References}

1. Balldin G, Borgström A, Genell S and Ohlsson K: The effect of peritoneal lavage and aprotinin in the treatment of severe acute pancreatitis. Res Exp Med (Berl) 183: 203-213, 1983.

2. Yu G, Wan R, Yin G, Xiong J, Hu Y, Xing M, Cang X, Fan Y, Xiao W, Qiu L, et al: Diosmetin ameliorates the severity of cerulein-induced acute pancreatitis in mice by inhibiting the activation of the nuclear factor- $\kappa \mathrm{B}$. Int J Clin Exp Pathol 7: 2133-2142, 2014.

3. Xiao WQ, Yin GJ, Fan YT, Qiu L, Cang XF, Yu G, Hu YL, Xing $\mathrm{M}$, Wu de Q, Wang XP, et al: Catalpol ameliorates sodium taurocholate-induced acute pancreatitis in rats via inhibiting activation of nuclear factor kappa B. Int J Mol Sci 15: 11957-11972, 2014.

4. Malleo G, Mazzon E, Siriwardena AK and Cuzzocrea S: TNF-alpha as a therapeutic target in acute pancreatitis - lessons from experimental models. ScientificWorldJournal 7: 431-448, 2007.

5. Li F, Zhang H, Xu KY, Wei Q and Zhou GX: Role of the chemokine fractalkine in a rat model of acute necrotizing pancreatitis and the interventional effect of ulinastatin. Arch Iran Med 16: 83-87, 2013.

6. Inoue K, Takano H, Shimada A, et al: Urinary trypsin inhibitor protects against systemic inflammation induced by lipopolysaccharide. Mol Pharmacol 67: 673-680, 2005.

7. Feng C, Su X, Chen LI, Zhou X, Li B, Wang LL, Lv FQ and Li TS: Ulinastatin enhances the therapeutic effect of intraperitoneal lavage on severe acute pancreatitis in rats. Exp Ther Med 9: 1651-1655, 2015.

8. Leonhardt U, Seidensticker F, Fussek M, Stockmann F and Creutzfeldt W: Camostate (FOY-305) improves the therapeutic effect of peritoneal lavage on taurocholate induced pancreatitis. Gut 31: 934-937, 1990.

9. Bhatia M, Wong FL, Cao Y, et al: Pathophysiology of acute pancreatitis. Pancreatology 5: 132-144, 2005.

10. Ohlsson $\mathrm{K}$ and Tegner $\mathrm{H}$ : Experimental pancreatitis in the dog. Demonstration of trypsin in ascitic fluid, lymph and plasma. Scand J Gastroenterol 8: 129-133, 1973.

11. Wang G, Wen J, Wilbur RR, Wen P, Zhou SF and Xiao X: The effect of somatostatin, ulinastatin and Salvia miltiorrhiza on severe acute pancreatitis treatment. Am J Med Sci 346: 371-376, 2013.

12. Wallner G, Solecki M, Ziemiakowicz R, Ćwik G, Dyndor P and Maciejewski R: Morphological changes of the pancreas in course of acute pancreatitis during treatment with Ulinastatin. Pol Przegl Chir 85: 114-122, 2013.

13. Matsukawa $\mathrm{H}$, Hara $\mathrm{A}$, Ito $\mathrm{T}$, et al: Continuous arterial infusion of protease inhibitor with supplementary therapy for the patients with severe acute pancreatitis - clinical effect of arterial injection of ulinastatin. Nihon Shokakibyo Gakkai Zasshi 95: 1229-1234, 1998 (In Japanese).

14. Fisic E, Poropat G, Bilic-Zulle L, Licul V, Milic S and Stimac D: The role of IL-6, 8 and 10, sTNFr, CRP and pancreatic elastase in the prediction of systemic complications in patients with acute pancreatitis. Gastroenterol Res Pract 2013: 282645, 2013.

15. Papachristou GI: Prediction of severe acute pancreatitis: Current knowledge and novel insights. World J Gastroenterol 14: 6273-6275, 2008.

16. Zhang XP, Zhang L, Chen LJ, et al: Influence of dexamethasone on inflammatory mediators and NF-kappaB expression in multiple organs of rats with severe acute pancreatitis. World J Gastroenterol 13: 548-556, 2007.

17. Schäfer C, Tietz AB and Göke B: Pathophysiology of acute experimental pancreatitis: lessons from genetically engineered animal models and new molecular approaches. Digestion 71 : 162-172, 2005.

18. O'Reilly DA, Roberts JR, Cartmell MT, Demaine AG and Kingsnorth AN: Heat shock factor-1 and nuclear factor-kappaB are systemically activated in human acute pancreatitis. JOP 7: 174-184, 2006.

19. Algül H, Tando Y, Schneider G, Weidenbach H, Adler G and Schmid RM: Acute experimental pancreatitis and NF-kappaB/Rel activation. Pancreatology 2: 503-509, 2002. 